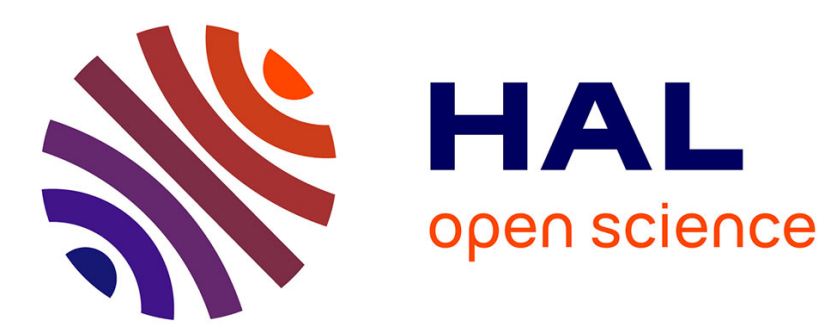

\title{
Group independent component analysis of resting state EEG in large normative samples
}

Marco Congedo, Roy John, Dirk de Ridder, Leslie Prichep

\section{To cite this version:}

Marco Congedo, Roy John, Dirk de Ridder, Leslie Prichep. Group independent component analysis of resting state EEG in large normative samples. International Journal of Psychophysiology, 2010, 78, pp.89-99. 10.1016/j.ijpsycho.2010.06.003 . hal-00523200

\section{HAL Id: hal-00523200 \\ https://hal.science/hal-00523200}

Submitted on 4 Oct 2010

HAL is a multi-disciplinary open access archive for the deposit and dissemination of scientific research documents, whether they are published or not. The documents may come from teaching and research institutions in France or abroad, or from public or private research centers.
L'archive ouverte pluridisciplinaire HAL, est destinée au dépôt et à la diffusion de documents scientifiques de niveau recherche, publiés ou non, émanant des établissements d'enseignement et de recherche français ou étrangers, des laboratoires publics ou privés. 
EEG RSN by Group ICA - Congedo et al. 2010

\title{
Group independent component analysis of resting-state EEG in large normative samples
}

\author{
Marco Congedo ${ }^{\mathrm{a} *}$, Roy E. John ${ }^{\mathrm{b}, \mathrm{c}}$, Dirk De Ridder ${ }^{\mathrm{d}}$, Leslie Prichep ${ }^{\mathrm{b}, \mathrm{c}}$ \\ a Team ViBS (Vision and Brain Signal processing), Gipsa-lab, National Center for Scientific Reserach (cnrs), \\ Grenoble University, Grenoble, France.
}

b Brain Research Laboratory, New York University Medical School, Department of Psychiatry., New York, USA

c Nathan S. Kline Institute for Psychiatric Research, Orangeburg, New York, USA

d Brain Research center Antwerp for Innovative and Interdisciplinary Neuromodulation $\left(B R A I^{2} N\right) \&$ Dept of Neurosurgery, University and Hospital of Antwerp, Belgium

\footnotetext{
* Corresponding author: Gipsa-lab. 961 rue de la Houille Blanche - Domaine universitaire - BP 46 38402, Grenoble, France.

Tel: + $33(0) 476826252$; Fax: + $33(0) 476574790$

e-mail: Marco.Congedo@gmail.com
}

Authors' copy. Accepted for publication in the "International Journal of Psychophysiology"

\section{Keywords:}

Electroencephalography (EEG), Resting State, Default Mode, Blind Source Separation (BSS), Independent Component Analysis (ICA), Norms, Inverse Solution, Source Localization. 


\section{ABSTRACT}

EEG (Electroencephalography) resting state were studied by means of group blind source separation (gBSS), employing a test-retest strategy in two large-sample normative databases $(\mathrm{N}=57$ and $\mathrm{N}=84)$. Using a BSS method in the complex Fourier domain and a model-driven distributed inverse solution we closely replicate both the spatial distribution and spectral pattern of seven source components. Norms were then constructed for their spectral power so as to allow testing patients against the norms. As compared to existing normative databases based on scalp spectral measures, the resulting tool defines a smaller number of features with very little intercorrelation. Furthermore, these features are physiological meaningful as they relate the activity of several brain regions, forming a total of seven patterns, each with a peculiar spatial distribution and spectral profile. This new tool, that we name normative independent component analysis (NICA), may serve as an adjunct to diagnosis and assessment of abnormal brain functioning and aid in research on normal resting-state networks. 


\section{INTRODUCTION}

Over two decades ago it was demonstrated that the resting state of the brain in normal individuals is characterized by spectral features that could be reliably described by a series of mathematical equations as a function of age across the human life span (6-90 years). Patients with psychiatric and neurological conditions show significant deviations from such spectral patterns, (Ahn et al. 1980; John et al. 1977, 1980a, 1980b, 1987, 1988). These norms have been shown to be culture-fair and replicable, with high sensitivity and specificity to neuropsychiatric disorders (Kondacs and Szabo, 1999; Prichep, 2005; Hughes \& John, 1999; Coburn 2006). It has been theorized that this baseline or "ground state" of the brain results from a complex homeostatic system regulated by neurotransmitters and exists as a property of the resonant systems of the brain (John and Prichep, 2009).

For several reasons EEG is a suitable tool for building and using large-sample normative databases. First, modern EEG equipment is small, light and economical; EEG can be safely recorded on individuals of any age (including premature newborns), in any condition (even if extremely disabling such as profound states of unconsciousness), and virtually anywhere (e.g., at the bed of an intensive care unit, in the incubator, on top of a mountain, etc.). Second, given enough data are averaged, EEG spectral measures of the same individual are highly reliable over months and even years (Fein et al. 1984; Kondacs and Szabo, 1999). EEG spectral features stabilize after a few handfuls of seconds, with as few as one minute of artifact-free EEG yielding reliable spectral measures (Nunez and Srinivasan, 2006; Oken and Chiappa, 1988). Such reliability is better verified in EEG continuously recorded during a resting state with the eyes closed and for relative power measures (John et al. 1987). Such stability is now also reported with PET (although norms still do not exist), but not with fMRI (Raichle and Snyder, 2007). Third, consistent EEG norms have been found across cultures and ethnicities, as verified comparing independent studies from a multitude of countries (Hughes and John, 1999; Prichep 2005). It is accepted that the independence of the EEG spectrum from cultural and ethnic factors reflects the common genetic heritage of the mankind. For example, a study on a large sample of 16-year-old twins found that the variance of EEG power is mostly (76\% to $89 \%$ depending on the frequency band) explained by heritability (van Beijsterveldt et al. 1996). In summary, intra-subject reliability, inter-subject consistence and the ease of 
recording procedures can be considered the fundamental properties of EEG enabling quantitative assessment of brain integrity in persons of any age, origin or background through comparison to normative values.

The interest for brain function in resting state has recently re-gained considerable interest with the advent of PET (positron emission tomography) and fMRI (functional magnetic resonance imaging). We now know that on average the human brain extracts about $40 \%$ of available oxygen in the blood and disposes about $20 \%$ of the energy for the whole body. Still, it amounts to only $2 \%$ of the total body weight. A considerable amount of this energy is consumed when the brain is at rest. On the other hand, it is has been estimated that the local task-related increase of energy consumption relative to the baseline is less then 5\% (Fox and Raichle, 2007; Raichle and Mintum, 2006). It is still unclear why the brain requires such an important supply of energy in the absence of targeted goals, but these observations have prompted a new wave of investigation on the "baseline" (ground level) brain functions. The functional organization of the brain at rest is currently conceived in terms of resting state networks (RSN), clusters of brain regions, mostly cortical, inter-connected anatomically and functionally. The study of RSNs has shifted the focus in neuroimaging from the exact localization of specialized brain functions (looking for "things in a place") to the understanding of the interplay of widespread brain structures (identifying networks). A consistent finding is that regions in the brain with similar functionality tend to be correlated in their spontaneous activity (Fox and Raichle, 2007). Several of such RSNs have been identified by PET and fMRI, relating to the motor, auditory and visual system, language, memory, dorsal and ventral attention and the default mode (Auer, 2008; Beckmann et al. 2005; Damoiseaux et al. 2006; Fox et al. 2005, 2006; Fox and Raichle, 2007; Fransson, 2005; Mantini et al. 2007; van den Heuvel et al. 2008). The default mode network (DMN), appears the most active RSN at rest (for a review see Auer, 2008; Broyd et al. 2008; Buckner et al. 2008; Fox and Raichle, 2007), thus it is putatively the most energy-demanding brain function of all. As for EEG spectral measures, the DMN appears to have a counterpart in primates (Rilling et al. 2007; Vincent et al. 2007) and to develop with age (Bluhm et al. 2008; Fair et al. 2007), while clinical studies accumulate evidence on the alteration of the DMN in Attention Deficit Disorder (Castellanos et al. 2008; Uddin et al. 2008), Alzheimer's disease (Greicius et al. 2004; Rombouts et al. 2005; Sorg et al. 2007), autism (Kennedy et al. 2006), chronic pain (Baliki et al. 2008), epilepsy (Laufs et al. 2007) and schizophrenia (Garrity et al. 2007). That is to say, as for EEG spectral norms discussed above, the DMN is not a mere epiphenomenon, 
but an essential ingredient of the healthy brain functioning, peculiar to the phylogenetic and ontogenetic evolution of the mankind.

Two main data analysis approaches have been used to study functional connectivity in resting state networks by fMRI (Buckner et al. 2008. For a comparison see Bluhm et al. 2008): a seed-based connectivity analysis and independent component analysis (ICA). The latter is currently enjoying increasing popularity thanks to its complete data-driven nature (Beckmann et al. 2005; Bluhm et al. 2008; Greicius et al. 2004; Eichele et al. 2008; Mantini et al. 2007; Scheeringa et al. 2008). Regarding EEG, biophysical and neurophysiological studies suggest that each resting state pattern may exhibit complex dynamics unfolding over time with multiple frequencies (Jann et al. 2009; Mantini et al. 2007). The mass of recent literature suggests that checking univariate power measurements (at each electrode separately) may not be the best methodology for studying the resting brain, (see concerns expressed by Jann et al. 2009). Studying the distribution of scalp EEG power at rest, as in the aforementioned studies on EEG normative database or more recently in Chen et al. (2008), does not allow the study of baseline patterns because scalp voltage is a mixing of underlying source activity (volume conduction: see Nunez and Srinivasan, 2006) and because scalp power is an appropriate measure of local neuronal synchronization, not of widespread coherent synchronization. Instead, we aim at extracting spatial maps of widespread synchronizations over the cortex, which can be treated as a single phenomenon and can be tested altogether (the whole cohort) along the frequency dimension. Besides allowing the study of co-activation of several brain areas, such approach allows the standardization of the spatial extent of the activations across individuals and also a smaller number of features (data compression) with lower intercorrelation (reduction of volume conduction effects). With fMRI such investigation in large samples of individuals has been performed by group ICA (Calhoun et al. 2001; Schmithorst and Holland, 2004), an approach that we introduce here in the context of EEG. This approach is essentially different from the seedbased approach in that we do not need to define explicitly measures of synchronization between different brain areas. This is particularly advantageous in the context of EEG, since many of such measures are influenced by the effect of volume conduction (Nunez and Srinivasan, 2006). 
In this paper we describe the Normative Independent Component Analysis (NICA). Using this method we describe the extraction of eyes-closed resting EEG patterns using group ICA and the norming of the components thus extracted. ICA extracts scalp spatial maps and associated EEG time-courses, referred together to as components (Makeig et al. 2004). Since there is no way to establish a-priori how many of such components should be extracted nor if they are reliable, we employ a test-retest strategy using two independent large sample normative databases $(\mathrm{N}=57$ and $\mathrm{N}=84)$ and retain only replicable components; such strategy has been previously employed by Damoiseaux et al. (2006) in an fMRI study. Once robust ICA normative components are extracted, we characterize the cortical structures involved in each component using source localization, sLORETA (Pascual-Marqui, 2002), a model-driven distributed inverse solution of the components spatial maps (Greenblatt et al. 2005; Lopes da Silva, 2004) and their associated spectral profile. In the two databases, we describe seven replicable components with nearly identical spatial distribution and spectral profile. These components are then normed and patients tested against the normative values for each component.

\section{METHOD AND RESULTS}

\section{Subjects and EEG recording procedures}

In order to avoid age effects we consider in this study only adult individuals between 17 and 30 years of age. Two independent normative databases previously acquired were used for this study. One is a subset of the normative database of the Brain Research Laboratory (BRL), New York University School of Medicine (N=57; age range 17-30) and the other the normative database of Nova Tech EEG (NTE), Inc., Mesa, AZ (N=84; age range 18-30). Exclusion criteria for the BRL database were known psychiatric or neurological illness, history of drug/alcohol abuse, current psychotropic/CNS active medications, history of head injury (with loss of consciousness) or seizure disorder. Exclusion criteria for the NTE database were a psychiatric history in any relative and participant of drug/alcohol abuse, head injury (at any age, even very mild), headache, physical disability and epilepsy.

Recording procedures and settings were very similar for the two databases. In both cases 3-20 minutes of EEG data was continuously recorded while the participant sat with the eye-closed on a comfortable chair in a 
quiet and dimly lit room. EEG data were acquired from the 19 standard locations prescribed by the 10-20 International System (Jasper, 1958: FP1, FP2, F7, F3, FZ, F4, F8, T3, C3, CZ, C4, T4, T5, P3, PZ, P4, T6, O1, O2) using linked ear reference and enabling a $60 \mathrm{~Hz}$ notch filter to suppress power line contamination. The impedance of all electrodes was kept below 5K Ohms. Data of the NTE database were acquired using the 12-bit A/D NeuroSearch-24 acquisition system (Lexicor Medical technology, Inc., Boulder, CO) and sampled at 128 $\mathrm{Hz}$, whereas data of the BRL database were acquired using the 12-bit A/D BSA acquisition system (Neurometrics, Inc., New York, NY) and sampled at $100 \mathrm{~Hz}$. For consistency, we subsequently up-sampled the BRL database to $128 \mathrm{~Hz}$ using a natural cubic spline interpolation routine (Congedo et al. 2002). In order to minimize inter-subject variability we removed from all data any biological, instrumental and environmental artifacts, paying particular attention to biological artifacts generated by the eyes, the hearth and the muscles of the neck, face and jaw. The recordings of the two databases had been carefully screened by one EEG technician and one experienced EEG researcher. Additionally, author MC reviewed all recordings. All recordings included in this study feature high overall signal-to-noise ratio. The mean length and standard deviation of artifact-free data in the BRL $(\mathrm{N}=57)$ and NTE $(\mathrm{N}=84)$ database were 102.9 (27.5) and 92.5 (29.79) seconds, respectively.

\section{Frequency Domain Statistics}

All statistics used in this study are summarized in the complex Hermitian Fourier cross-spectral matrices $\boldsymbol{S}_{f} \in \mathbb{C}^{E \cdot E}$, where $f$ is the discrete frequency index and $E$ the number of electrodes (Bloomfield, 2000; Brillinger, 1975). We can write

$$
\boldsymbol{S}_{f}=\boldsymbol{C}_{f}+i \boldsymbol{Q}_{f}
$$

where $i=\sqrt{-1}$. Real symmetric $C_{f} \in \mathfrak{R}^{E \cdot E}$, referred to as the cospectral matrix, holds in the main diagonal the power spectra and in the off-diagonal elements the in-phase (or with a half cycle phase shift, i.e., opposite sign) dependency structure. Imaginary antisymmetric $i \boldsymbol{Q}_{f} \in \mathfrak{I}^{E \cdot E}$, referred to as the quadrature spectral matrix, holds in the off-diagonal elements the out-of-phase (a quarter cycle in either direction) dependency structure. The cospectral matrix is equivalent to the covariance matrix of the data band-pass filtered for its discrete 
frequency: by Parseval's theorem the sum of all co-spectral matrices is equivalent to the data covariance matrix. We estimate individual cross-spectral matrices as the average obtained by Fast Fourier Transform (FFT) on 50\% sliding overlapping 2-seconds windows tapered using the function introduced by Welch (1967). With $k: 1, \ldots, K$ indicating the $k^{\text {th }}$ subject in the normative database, let us denote $\boldsymbol{C}_{k f}^{\text {abs }}$ the estimated cospectral matrix for subject $k$ at discrete frequency $f$. Here "abs" reminds that in the literature such statistics are referred to as "absolute power". We will also make use of "relative power" estimations, given by

$\boldsymbol{C}_{k f}^{\mathrm{rel}}=\boldsymbol{C}_{k f}^{\mathrm{abs}} / \sum_{f=1}^{F} \operatorname{tr}\left(\boldsymbol{C}_{k f}^{\mathrm{abs}}\right)$

where "tr" indicates the trace of the argument. Those are the cospectral matrices normalized by the total power in the whole frequency range of interest. Finally, we will use "normal power" cospectra defined such as

$\boldsymbol{C}_{k f}^{\mathrm{nor}}=\boldsymbol{C}_{k f}^{\mathrm{abs}} / \operatorname{tr}\left(\boldsymbol{C}_{k f}^{\mathrm{abs}}\right)$

that is, the set where all cospectra are normalized by the total power in that discrete frequency. Absolute and relative power estimations are a long-standing standards in quantitative EEG analysis. Here we introduce also normal power estimations, which usefulness will appear in the sequel. The diagonal elements of normal power cospectra indicate for each frequency the proportion of power expressed by each channel with respect to all of them.

\section{Group Independent Component Analysis}

In both fMRI and EEG ICA has become a major processing tool, although the subjacent data-generative model and consequent interpretation of results is substantial different in the two cases. In the case of EEG the use of ICA has a clear and solid physiological interpretation within the context of the volume conduction theory; dipolar fields produced by collinear pyramidal cell columns in extended neocortical region results in mixed (superimposed) potential at each scalp electrode. The current diffusion is instantaneous and linear with good approximation (for a review see Congedo et al. 2008). In essence, we use the recorded time-series of scalp potentials to estimate the underlying dipolar fields. For $E$ scalp sensors and $M \leq E$ EEG dipolar fields to be estimated, the linear model employed describes the superposition principle, i.e., we state

$\boldsymbol{v}(t)=\boldsymbol{A s}(t)$, 
where $\boldsymbol{v}(t) \in \mathbb{R}^{E}$ is the sensor measurement vector, $\boldsymbol{A} \in \mathbb{R}^{E \cdot M}$ is a time-invariant non-singular mixing matrix and $\boldsymbol{s}(t) \in \mathbb{R}^{M}$ holds the time-course of the source components. Note that model (4) describes the instantaneous (in-phase) diffusion of current source over measurement sites, in fact describing the effect of primary current and volume conduction (Congedo et al. 2008). Our source estimation is given by

$\hat{\boldsymbol{s}}(t)=\boldsymbol{B} \boldsymbol{v}(t)$,

where $\boldsymbol{B} \in \mathbb{R}^{M \cdot E}$ is called the demixing or separating matrix. The $m^{\text {th }}$ row of $\boldsymbol{B}$ is a spatial filter holding coefficients for each electrode recovering the $m^{\text {th }}$ source component, the time-series of the dipolar field estimated by an inverse process. The $m^{\text {th }}$ column of $\boldsymbol{A}=\boldsymbol{B}^{+}$, with superscript + indicating the Moore-Penrose pseudo-inverse, is the estimation of the scalp spatial pattern associated with the $m^{\text {th }}$ source.

A wide array of ICA methods exist. In this work we use a method based on the approximate joint diagonalization (AJD) of Fourier cospectral matrices, which is a robust and computationally fast approach (Congedo et al. 2008). We diagonalize cospectral matrices in the frequency range $0.5-30 \mathrm{~Hz}$ only, which is the range with highest signal-to-noise ratio subsequently analyzed. As reported by Makeig et al. (2004), BSS of EEG appears to give satisfactory results within this frequency band-pass region. This results in $F=60$ frequencies with $0.5 \mathrm{~Hz}$ resolution $(0.5,1,1.5, \ldots, 30 \mathrm{~Hz})$. We perform ICA as the AJD of the grand-average Fourier cospectral matrices, which is an approach to group ICA analogous to the averaging group ICA approach described for fMRI by Schmithorst and Holland (2004).

In order to estimate $M<E$ source components the matrix $\boldsymbol{B}$ is found with a classical two-stage process, which allows the estimation of the $M$ most energetic components while reducing the noise. First the size of all input cospectra is reduced by transformation

$\overline{\boldsymbol{D}}_{f}^{\text {nor }} \in \mathbb{R}^{M \cdot M}=\boldsymbol{F} \overline{\boldsymbol{C}}_{f}^{\text {nor }} \boldsymbol{F}^{T}$, for all $f: 1, \ldots, F$,

where

$\overline{\boldsymbol{C}}_{f}^{\mathrm{nor}}=\frac{1}{K} \sum_{k=1}^{K} \boldsymbol{C}_{k f}^{\text {nor }}$ 
is the grand-average of the individual normal cospectral matrices $(3)^{1}$. Matrix

$\boldsymbol{F} \in \mathbb{R}^{E \cdot M}=\left(\sum_{f=1}^{F} \overline{\boldsymbol{C}}_{f}^{\mathrm{nor}}\right)^{-1 / 2}$

is often referred to as the "whitening" or "sphering" matrix, since definition (8) implies

$\boldsymbol{F} \sum_{f=1}^{F} \overline{\boldsymbol{C}}_{f}^{\mathrm{nor}} \boldsymbol{F}^{T}=\boldsymbol{I} \in \mathbb{R}^{M \cdot M}$,

where $\boldsymbol{I}$ is the identity matrix. In (7) and (8) we sum across frequencies in the diagonalization range, that is, 0.5 $30 \mathrm{~Hz}$. In this high signal-to-noise range the multidimensional variance structure of the data in the two databases is very similar. We analyzed the eigenvalues of the grand-average cospectral matrix summed across analyzed frequencies. Figure 1 shows the mean square representation error (MSRE), the complement of the cumulative normalized sum of the eigenvalues, and the Akaike Information criterion (Wax and Kailath, 1985). Both functions have very similar shapes in the two databases. The mimimum AIC is reached in four orthogonal dimensions for both databases.

Insert Fig 1 Approximately Here

Once obtained cospectral matrix $\overline{\boldsymbol{D}}_{f}^{\text {nor }}$ in the reduced $M$-dimensional space the approximate joint diagonalization (AJD) of the transformed set is performed. AJD is a powerful algebraic method seeking a matrix $\boldsymbol{R} \in \mathbb{R}^{M \cdot M}$ such that products $\boldsymbol{R} \overline{\boldsymbol{D}}_{f}^{n o r} \boldsymbol{R}^{T}$ are as diagonal as possible for all $f$. Since the off-diagonal elements of the cospectra hold statistics describing the all pair-wise in-phase dependency between electrodes, the sought demixing matrix holds in rows spatial filters maximizing the in-phase independence of the sources computed as per (5). Finally, we obtain the solution to the ICA problem as

$\boldsymbol{B}=\boldsymbol{R} \boldsymbol{F} \in \mathbb{R}^{M \cdot E}$.

\footnotetext{
${ }^{1}$ Because of the individual global scaling factor seen in EEG (Hernández et al. 1994; Goncalves et al. 2006) we average individual normal cospectral matrices and not their absolute counterpart so that all individuals contribute fairly to the average.
} 
To solve the AJD problem, hence the ICA problem, we use an iterative algorithm previously developed (Congedo and Pham, 2009; Pham and Congedo, 2009). Such algorithm allows specifying weights for each input matrix. Following Congedo et al. (2008) we use a weighting function encouraging the diagonalization effort proportionally to the non-diagonality of the input matrices.

Note that we are using only second order statistics, thus we are not assuming complete zero-lag independence of the source processes, but only their uncorrelatedness. While we can relax the assumption of independence, an additional assumption on the sources is necessary for achieving their separation. Using AJD of a set of cospectral matrices, such additional condition is that the source components have non-proportional spectral profile (Pham, 2001). We feel that such an assumption is appropriate for this study since it is physiologically unlikely that different components operate with exactly the same frequencies along the whole frequency spectrum of interest. To better fulfill the assumption, however, we have used normal cospectral matrices (7) as input to the AJD algorithm. As we will show the normal power spectral profile are less proportional than the absolute and relative counterparts. For more details on the implementation of this ICA approach and for a review of the physical and statistical background of its use in the context of EEG the reader is referred to the review by Congedo et al. (2008).

\section{Distributed source localization}

The previous sub-section showed how we obtained the spatial filters $\boldsymbol{B}$. The estimated mixing matrix of model (4) is $\boldsymbol{A}=\boldsymbol{B}^{+}$, which using (8) and (9) is

$$
\boldsymbol{A}=(\boldsymbol{R} \boldsymbol{F})^{+}=\left(\sum_{f=1}^{F} \overline{\boldsymbol{C}}_{f}^{\mathrm{nor}}\right)^{1 / 2} \boldsymbol{R}^{-1}
$$

The $m^{\text {th }}$ column of the estimated mixing matrix is the scalp spatial pattern (voltage) associated to the $m^{\text {th }}$ source component and can enter an inverse solution method for estimating its cortical location, just as EEG samples. For this purpose we use the popular standardized low resolution electromagnetic tomography (sLORETA) 
method (Pascual-Marqui, 2002), which has received theoretical support (Greenblatt et al. 2005; Pascual-Marqui, 2007) and has been validated independently in several laboratories (Congedo, 2006; Terakawa et al. 2008; van der Loo et al. 2009; Wagner et al. 2004). sLORETA is a smooth model-driven distributed inverse solution, implying that no a-priori knowledge of the number of active dipoles for each component is required. The only user-adjustable parameter of the model-driven sLORETA method is the amount of Tikhonov regularization. Since the number of electrode is low, the mixing matrix is estimated on a large sample and the ICA spatial patterns we extract have higher signal-to-noise ratio as compared to raw EEG samples, we do not need regularization (Congedo, 2006). For computing sLORETA current density and plotting the results we used the freeware sLORETA-key software ${ }^{2}$.

\section{Feature extraction.}

Once obtained the demixing matrix $\boldsymbol{B}$ for the normative sample we can estimate the distribution of the spectral power for each source at each discrete frequency. Taking the expectation of the variance (spectral power) of (5) the component absolute power for the $k^{\text {th }}$ subject, $m^{\text {th }}$ source and $f^{\text {th }}$ discrete frequency is given by quadratic form

$\eta_{k m f}^{\mathrm{abs}}=\boldsymbol{b}_{m}^{T} \boldsymbol{C}_{k f}^{\mathrm{abs}} \boldsymbol{b}_{m}$

Similarly, the relative power is given by

$$
\eta_{k m f}^{\mathrm{rel}}=\boldsymbol{b}_{m}^{T} \boldsymbol{C}_{k f}^{\mathrm{rel}} \boldsymbol{b}_{m}
$$

and the normal power by

$$
\eta_{k m f}^{\mathrm{nor}}=\boldsymbol{b}_{m}^{T} \boldsymbol{C}_{k f}^{\mathrm{nor}} \boldsymbol{b}_{m}
$$

On the other hand, the corresponding features for the traditional scalp norms are the spectral power of the signal recorded at the measurement sites, i.e., the diagonal elements of $\boldsymbol{C}_{k f}^{\text {abs }}, \boldsymbol{C}_{k f}^{\text {rel }}$ and $\boldsymbol{C}_{k f}^{\text {nor }}$. In this case $M=E$, that is,

\footnotetext{
${ }^{2}$ This software implements revisited realistic electrode coordinates (Jurcak et al. 2007) and the lead field produced by Fuchs et al. (2002) applying the boundary element method on the MNI-152 (Montreal neurological institute, Canada) template of Mazziotta et al. (2001). The sLORETA-key anatomical template divides and label the neocortical (including hippocampus and anterior cingulated cortex) MNI-152 volume in 6239 voxels of dimension $5 \mathrm{~mm}^{3}$, based on probabilities returned by the Demon Atlas (Lancaster et al. 2000). The coregistration makes use of the correct translation from the MNI-152 space into the Talaiach and Tournoux (1988) space (Brett et al. 2002). LORETA-Key is freely available at URL http://www.uzh.ch/keyinst/loreta.htm.
} 
there are as many "spatial filters" as electrodes. Equivalently, such features can be obtained using $\boldsymbol{i}_{m}$ instead of $\boldsymbol{b}_{m}$ in (11-13), where $\boldsymbol{i}_{m}$ is the $m^{\text {th }}$ column of the identity matrix. This manipulation shows the essential difference between scalp EEG norms and NICA; the former focuses on EEG power at the electrode sites while the latter seeks linear combinations of such measures (with coefficient given by the $\boldsymbol{b}_{m}$ vectors). It follows that NICA uses the electrode sites altogether for deriving each feature, thus allowing the full spatial characterization of all features. Since our NICA method is specifically designed to minimize the volume conduction, the features we obtain are as little correlated as possible.

\section{Deriving norms and assessing normality of patients.}

Using equations (11-13) above we obtain features $\eta_{k m f}$ for each individual in the database, for absolute, relative and normal power. Norms are the central tendency and confidence intervals of their distribution. The lower and upper limits are chosen so as to include $(1-\alpha) \%$ of the distribution around the central tendency, where $\alpha$ is the type I error tolerated (false positive rate). For a given patient to be tested against the database, hereafter denoted by subscript $q$, we compute the corresponding features as $\chi_{q m f}^{\mathrm{abs}}=\boldsymbol{b}_{m}^{T} \boldsymbol{C}_{q f}^{\mathrm{abs}} \boldsymbol{b}_{m}, \quad \chi_{q m f}^{\mathrm{rel}}=\boldsymbol{b}_{m}^{T} \boldsymbol{C}_{q f}^{\mathrm{rel}} \boldsymbol{b}_{m}$ and $\chi_{q m f}^{\text {nor }}=\boldsymbol{b}_{m}^{T} \boldsymbol{C}_{q f}^{\text {nor }} \boldsymbol{b}_{m}$ for absolute, relative and normal power, respectively. Values of $\chi_{q m f}$ falling on the left of the lower limit flag a deficit of energy, whereas values falling on the right of the upper limit flag an excess of energy. Since the distribution of power measurements in not Gaussian, one may log-transform them in order to approximate Gaussianity and then obtain the limits as the mean value $\pm(\zeta \cdot$ standard deviation), where $\zeta$ is a real number depending on $\alpha$, e.g., 1.96 for $\alpha=0.05$ (John et al. 1987). In Congedo and Lubar (2003) it was shown that the accuracy of such method critically depends on the approximation to Gaussianity achieved by the transformation and that using this method the rate of false positive and false negative is not uniform across features. We use instead the non-parametric method proposed in Congedo and Lubar (2003), which yield uniform errors. Let us sort in ascending order the $K$ values for each feature. Then let $\omega=\operatorname{int}(K \cdot \alpha / 2)$, where "int" returns the integer part of the argument; the lower limit is defined as the $\omega^{\text {th }}$ sorted feature value and the upper limit as the $(K-\omega)^{\text {th }}$ sorted feature value. As before, values of $\chi_{q m f}$ falling on the left of the lower limit flag a 
deficit of energy, whereas values falling on the right of the upper limit flag an excess of energy. In both cases they flag possible abnormal brain functioning.

\section{RESULTS}

\section{Group normative ICA components}

The group ICA was independently applied on the NTE and BRL normative database starting with fixing the $M$, the number of estimated components, to four, the number suggested by the Akaike's informationtheoretic criterion (Figure 1) and increasing $M$ until the spectral and frequency distribution of the components found on the two databases do not match anymore. Proceeding this way seven replicable ICA components were identified. Reducing the space to seven dimension by pre-whitening ( $M=7$, see eq. (6) and (8)) explains $92.1 \%$ and $92.8 \%$ of the total grand-average variance in the $0.5-30 \mathrm{~Hz}$ for the BRL and NTE database, respectively.

The estimated current source spatial distribution (sLORETA) and associated spectral profile (absolute, relative and normal power) are shown in Figure 2. The seven components have nearly identical spatial distribution and spectral profile in the two databases. The first six components (C1-C6) have maximal current density in the medial portion of the brain. On the other hand, C7 has bilateral maximal current density. All components have nearly symmetric current distribution, with the exception of $\mathrm{C} 3$, which extend more on the right hemisphere according to both databases. Table 1 reports for each component the anatomical regions displaying high-power local maxima.

The seven components for each database in Fig. 2 have been sorted according to the amount of residual grand-average EEG variance explained in the $0.5-40 \mathrm{~Hz}$ range (Congedo et al. 2008). Figure 3 shows the variance explained by each component as obtained on the two databases. Note that not only the output components are sorted identically for the two databases (Figure 2) ${ }^{3}$, but also that each component individually contributes a similar increase of explained variance in the two databases. Note also that $\mathrm{C}$, which spatial

\footnotetext{
${ }^{3}$ This is due to the fact that the cospectral structure along frequencies is very similar in the two databases (Fig. 1) and also to the fact that the whitening matrix (8), which by definition sort the components by explained variance, achieves joint diagonalization in fair approximation, which is refined by the ensuing AJD algorithm without changing the order of the components.
} 
distribution in the two database matches only roughly, explains less then $5 \%$ of the variance. This suggests that increasing $M$ over seven would require the estimation of components with disadvantageous signal-to-noise ratio. In fact, the spatial localization of C7 is already pretty incongruent in the two databases, although their frequency profile is very similar ${ }^{4}$.

Insert Fig 2 and 3 Approximately Here

\section{ICA Components}

The spectral and spatial distribution of the seven ICA components are described below:

Component $1(\mathrm{C} 1)$ : $\mathrm{C} 1$ is characterized by a prominent normal theta activity and low normal alpha activity (Fig. 2). In the source space normal power quantifies the proportion of power generated by each component as compared to all the others, that is to say, the theta peak displayed by $\mathrm{C} 1$ indicates that $\mathrm{C} 1$ generates much of the total theta power generated all over the brain. $\mathrm{C} 1$ forms a network consisting of the subgenual and dorsal anterior cingulate (BA 23/24/32/33/25), insula (BA 13), middle/superior frontal gyrus and paracentral lobule (BA 4/5/6), and parahippocampal gurus (BA 28/34/35/36) (Table 1).

Component 6 (C6): C6 is characterized by low normal alpha power and high delta and beta normal power. Spatially it forms a network consisting of the medial frontal/rectal gyrus/anterior cingulate (BA 11, 25), middle frontal gyrus (BA 11), inferior frontal gyrus (BA 47), parahippocampal gyrus (BA 28/34), and insula (BA 13) (Table 1)

Component 5 (C5): The frequency spectrum of component 5 is similar to C6 (Fig. 2). Spatially, C5 forms a network consisting of the anterior cingulate (BA 24/25/32), medial frontal gyrus (BA 32/9/10/11), rectal/orbital gyrus (BA 11/47), inferior frontal gyrus (BA 47), and parahippocampal gyrus (BA 28/34) (Table 1).

\footnotetext{
${ }^{4}$ This is due to the fact that we are estimating directly the demixing matrix $\boldsymbol{B}$, which a-posteriori inversion provides us with the estimation of the mixing matrix $\boldsymbol{A}$, and not vice-versa as done by some other ICA algorithms (Hyvärinen, Karhunen and Oja, 2001). Error in the estimation of $\boldsymbol{B}$ will therefore be amplified in the estimation of $\boldsymbol{A}$ (Tichavsky and Yeredor, 2009). Now, the former provides estimation of the spectral profile, which is similar for all components in both databases (thus we believe that it is the same component), while the latter provides the input for source localization methods.
} 
Component $3(\mathbf{C 3})$ : $\mathrm{C} 3$ is characterized by a clear alpha peak in both absolute and normal power. Among all components the normal power alpha peak of $\mathrm{C} 3$ is the most pronounced (Fig. 2). C3 forms a network consisting of the cuneus/precuneus/ (BA 30/31/7), right superior parietal lobule (BA 7), Posterior Cingulate (BA 30), lingual/parahippocampal gyrus (BA 18/19/30), right fusiform gyrus (BA 19), sparing the primary visual cortex (Fig. 2 and Table 1).

Component 2 (C2): C2 consists of cuneus/precuneus/ (BA 7/31/18/19/), post-central gyrus (BA 3/4/5), superior parietal and paracentral lobule (BA 5/7), posterior cingulate gyrus (BA 23/31) (Table 1). It predominantly oscillates at alpha processing speed, similarly to $\mathrm{C} 3$, to which partially overlaps also spatially, without the extension to the fusiform and parahippocampal area, but extending instead into BA 5 and 7.

Component 4 (C4): $\mathrm{C} 4$ has an alpha spectral peak and occipital localization which is typically noted in eyes closed EEG. In contrast to $\mathrm{C} 2$ and $\mathrm{C} 3$, the normal spectrum of $\mathrm{C} 4$ does not peak at $10 \mathrm{~Hz}$, instead is equally high in between about $10 \mathrm{~Hz}$ and $22 \mathrm{~Hz}$. $\mathrm{C} 4$ extends to the $\mathrm{PCC}$, reaching the precuneus.

Component 7 (C7): C7 forms a predominant right lateralized network consisting of the post-central gyrus (BA 1/2/3), supramarginal gyrus/inferior parietal lobule (BA 40), precentral gyrus (BA 6), cuneus/ precuneus (BA 17/18/19/31), middle occipital gyrus (BA 18), superior and middle temporal gyrus (BA 21/22/39/41), insula (BA 13), angular gyrus (BA 39).

\section{DISCUSSION}

It has been found that the frontal midline theta rhythm is generated in the dACC (dorsal anterior cingulate cortex: Onton, Delorme and Makeig, 2005), possibly alternating with the VMPFC (ventromedial prefrontal cortex: Asada et al. 1999), at the same anatomical region as sLORETA localizes C1. It has been established that frontal midline theta oscillations are involved in attentional processes (Inanaga 1998). Accordingly, the component best matching the mid-frontal scalp topography associated with the frontal theta 
rhythm was negatively correlated to the default mode network activity (Scheeringa et al. 2008), whereas in a mental arithmetic induced workload combined EEG-fMRI experiment, theta power increase was associated with activation of the ACC (Sammer et al. 2007). Part of the resting state $\mathrm{C} 1$ and the salience network described by fMRI overlap anatomically, i.e., the dorsal ACC extending into the anterior insulae (Seeley et al. 2007). The salience network extends to the dorsomedial thalamus and hypothalamus, sublenticular extended amygdala and ventral tegmental area. This salience network unites conflict monitoring, interoceptive-autonomic, and rewardprocessing centers (Seeley et al. 2007). Furthermore the right dACC-insula salience network has been described as a switch between the central executive frontoparietal network and the default mode (Sridharan et al. 2008). Whenever new information is presented, activity levels of the dACC reflect the salience of the new information for predicting future outcomes (Critchley 2005; Behrens et al. 2007), guiding optimal decision making in an uncertain world (Kennerley et al. 2006). The dorsal ACC is anatomically (Ghashghaei et al. 2007) and functionally heavily connected to the subgenual ACC (Margulies et al. 2007; Stein et al. 2007) as well as to the thalamus and brainstem (Mottaghy et al. 2006). The subgenual ACC (BA25) is functionally also connected to the parahippocampal gyrus (Kahn et al. 2008). Based on these evidences, C1 described by NICA EEG seems to relate to the salience of internal and external stimuli, by combining networks involved in arousal and intrinsic alertness, interoceptive awareness and motivation. $\mathrm{C} 1$ could therefore be considered an attentional network focusing on salient information. A combined EEG-fMRI study could verify that $\mathrm{C} 1$ is inversely related to the DMN.

Functional connectivity fMRI studies suggest that the pattern seen in C6 is similar to a connectivity pattern with BA 25 as seed (Margulies et al. 2007), whereas C1 connects functionally more to the dACC (BA 24) (Margulies et al. 2007). Anatomical studies show that the sequence of information transmission between the subgenual and dACC might be direct and indirect via the amygdala (Ghashghaei et al. 2007), with the output of BA 25 going to the amygdala and from there to the dACC. fMRI connectivity studies on the other hand demonstrate information transmission from the amygdala to BA 25 and from there to the dACC area in BA 24 (Stein et al. 2007). The subgenual ACC extending into the posterior orbitofrontal cortex (OFC) is associated with positive reward (Elliott et al. 2000; Rogers et al. 2004; Drevets et al. 2008). In animals, this region participates in an extended "visceromotor network" of structures that modulates autonomic/neuroendocrine 
responses and neurotransmitter transmission during the neural processing of reward, fear, and stress (Drevets et al. 2008). The C6 component could therefore be considered a reward driven autonomic arousal system.

The pregenual cingulate and parts of the OFC seen in $\mathrm{C} 5$ are implicated in the continuous representation of affective hedonic value (Rogers et al. 2004; Keedwell et al. 2005; Grabenhorst et al. 2008; Walter et al. 2009). Thus C5 can be considered an emotion/hedonic extension of C6, a reward driven autonomic and emotional arousal system.

The alpha oscillations reflected in $\mathrm{C} 3$ are most likely those generated in the thalamus independently of sensory afferences (Schreckenberger et al. 2004). Closing the eyes at rest engenders EEG alpha power increase at PCC (Posterior Cingulate Cortex)/anterior precuneus and occipital regions (Barry et al. 2007), in agreement with the spatial localization of these components.

The anterior precuneus involvement seen in C2 may represent that involved in self-centered mental imagery, during both personal past and personal future thinking (Abraham et al. 2008). Dissociations between the areas involved in personal past versus personal future thinking are located more along the medial parietal wall (Abraham et al. 2008). The posterior precuneus subserves successful episodic visual memory retrieval (Cavanna and Trimble 2006). The parietal-parahippocampal network is related to topographic (static) and topokinetic (dynamic) spatial self-related memory (Berthoz 1997). The medial parietal part of the secondary somatosensory cortex processes and constantly updates proprioceptive input (Richer et al. 1993; Pellijeff et al. 2006). Thus C2 and C3 could be considered networks related to self-centered temporal and spatial memory based on proprioceptive and vestibular input (C3)(eyes closed) with associated visual memory retrieval (C2).

The distribution of $\mathrm{C} 4$ is suggestive of the extended visual cortex activity found in the resting state (Wang et al. 2008), and this resting state visual activity can be modulated by top down effects, such as thoughts (Johnson et al. 2007). It has been suggested that this resting state activity in the visual areas is related to visual imagery (Wang et al. 2008), which is known to activate visual areas (Farah 1989; Svoboda et al. 2006) and is expected in a eyes closed condition. $\mathrm{C} 4$ could therefore be considered a resting state visual imagery network. 
Although in absolute term an alpha peak is observed, in C7 normal power steadily increases with frequency. The right sided supramarginal gyrus in humans is involved in the processing of vestibular information for head and body orientation in space (Stephan et al. 2005). Electrical stimulation on the right angular gyrus induces vestibular and complex somatosensory responses (Blanke et al. 2002), suggesting that the angular/supramarinal junction might be involved in vestibular somatosensory integration of body orientation in space. Electrical burst stimulation of the posterior part of the superior temporal gyrus has induced out of body experiences in a controlled way associated with metabolic alterations at the junction of the right angular and supramarginal gyrus (De Ridder et al. 2007), demonstrating its role in embodiment. C7 could therefore be related to multisensory integration of body experience in space.

In an attempt to link our findings with existing literature on RSN we suggest that NICA C1 describes an attentional network focusing on salient information, C2 and C3 could be considered networks related to selfcentered temporal and spatial memory based on proprioceptive and vestibular input (C3)(eyes closed) with associated visual memory retrieval (C2). $\mathrm{C} 4$ could be considered a resting state visual imagery network. The $\mathrm{C} 6$ could be considered a reward driven autonomic arousal system and the $\mathrm{C} 5$ can be considered an emotion/hedonic extension of C6, a reward driven autonomic and emotional arousal system. C7 could be related to multisensory integration of body orientation in space. Thus when we close our eyes we can hypothesize, based on the current literature and present findings, that C1, C5 and C6 keep our homeostatic, interoceptive and reward based autonomic and emotional arousal system online, $\mathrm{C} 2$ and $\mathrm{C} 3$ relate to our self-perceived orientation in space and time based on proprioception and vestibular input, $\mathrm{C} 7$ to the bodily representation of the self, while C4 is related to memory based visual imagery, which starts on the moment we close our eyes. These hypotheses are highly speculative at this time. Future research should substantiate them by experiments aiming at eliciting the mentioned functional systems, to which changes in the power of the corresponding NICA components should be observed.

As any other source separation method NICA decomposes the whole EEG in a number of elementary components, each one characterised by its time course (here analyzed in the frequency domain as power spectra) 
and spatial pattern, here used as input to source localization by sLORETA. The degree of confidence toward those two aspects is not the same; the time course is extracted directly from the data after source separation (Eq. 5), whereas source localization introduces a bias due to the ensuing inverse solution. The latter is subjected to two types of error: the approximation of the head model needed to derive the forward solution and the possible reconstruction error of the inverse solution itself. In this study we have relied on the average realistic head model of Fuchs et al. (2002), which has been applied for all subjects. A more accurate procedure would be to compute the individual realistic head model, project the individual current density maps to a standard average brain and then average the results. Unfortunately this was not possible in this study as the MRI of the subjects involved in the normative databases was not available. Nonetheless, the anatomical localization errors introduced by the use of an average head model should average out in a large-sample group analysis. As per the source localization error introduced by the inverse solution, we know that sLORETA is capable of reconstructing simple dipole configurations (Congedo, 2006; Pascual-Marqui, 2002; Wagner et at. 2004). The decomposition operated by the source separation effectively distributes the number of active dipoles across components; that's why an inverse solution after source separation is more trustworthy as compared to the same inverse solution performed on raw EEG data. The normative databases we have employed were recorded with 19 electrodes. While this is a rather low number, in Congedo (2006) we have shown by point spread function simulations that using an approximate head model sLORETA is capable of exact localization of single dipoles with as few as six electrodes. Increasing the number of electrodes it increases the spatial resolution, that is, the ability to resolve two closely-spaced dipoles, not the localization ability (given the spatial sampling of the scalp is appropriate). In this study we have found seven replicable resting-state components. One should keep in mind that this number is by no means an absolute reference; it would likely increase with the number of individuals in the database, the number of electrodes used to record EEG and the overall signal-to-noise ratio. Also, our results are not easily comparable with single-subject BSS studies, especially those performed with high-density recording, in which typically BSS disentangle dipolar fields in a higher number of components. In conclusion, we feel that the spatial localization of the seven NICA components can be regarded as a fairly good approximation. Keeping in mind the limitation of the method, further studies confirming and expanding the present results are warranted. 
The RSNs described by fMRI are not the same as the patterns found by ICA of resting state EEG activity. There might be multiple reasons for that. So far no clear relationship has been established between very slow frequency BOLD oscillations $(<0.1)$ and EEG oscillations. Available data is scattered and inconsistent: using simultaneous EEG and fMRI it was found that BOLD signal in the DMN correlates positively with EEG power in the beta band (17-23 Hz: Laufs et al. 2003), but negatively with EEG frontal midline theta power (3-8 Hz) (Scheeringa et al. 2008). Mantini et al. (2007) concluded that the DMN fMRI BOLD fluctuations positively correlate with both Alpha and Beta EEG power $(8-30 \mathrm{~Hz})$, but also with gamma power $(30-50 \mathrm{~Hz})$. The BOLD signal is correlated to evoked gamma oscillations in both the visual and auditory cortex (Foucher et al. 2003; Mukamel et al. 2005; Lachaux et al. 2007; Nir et al. 2007), and anticorrelated to theta, alpha and beta local field potentials in the auditory cortex (Mukamel et al. 2005), suggesting that the BOLD signal in general seems to be correlated best to gamma band activity. It appears that one EEG feature can correlate with different fMRI activation maps and a single resting state network may also be associated with a variety of EEG patterns (Laufs 2008). Furthermore the fMRI resting state analyzes brain activity only indirectly via changes in blood oxygenation in contrast to EEG. Also the fMRI resting state evaluates neural activity at very different time dimensions, in the scale of 10-15 seconds. Synchronized low-frequency BOLD fluctuations likely arise from a combination of vascular low-pass filtering and low-frequency amplitude modulation of neural activity. This very complicated interaction between low-frequency BOLD fluctuations and brain physiology has resulted in criticism doubting that «this 'intrinsic' activity, although interesting, has any special significance » (Morcom and Fletcher 2007). The authors also state that «there may be some consistency in the network of regions active in the resting task, but without a theoretical synthesis that also identifies this as a qualitatively and quantitatively important baseline state, the idea of a default mode has little utility » (Morcom and Fletcher 2007).

The group ICA of resting state EEG appears to delineate small independent networks of anatomically contingent areas. How these independent small functional components interact to form larger networks, potentially similar to the ones described by fMRI functional connectivity, can be studied by out-of-phase coherence of the extracted components. Using the same two databases analyzed in the present study we have shown the organization of the seven components in two networks, where within each network components oscillate coherently with multiple-frequency dynamics (Congedo et al. 2010). 
Understanding the physiological role of the NICA independent components will allow a better understanding of pathologies by comparing ICA of pathological resting states to the described normative databases. Results in this direction obtained in a study on obsessive-compulsive disorder will be published elsewhere (Koprivova et al., submitted; De Ridder et al. in preparation). Our current and future research efforts aim at establishing the clinical utility and validity of NICA. For this purpose we have developed an executable application for performing NICA analysis. The application and the code are available to peers under request to the corresponding author.

\section{CONCLUSION}

The study of the brain at rest has a long-lasting tradition and has recently gained a renewed impulse thanks to the advent of modern neuroimaging modalities such as PET and fMRI. EEG normative databases built on large-samples have proven to be a useful adjunct in the diagnosis and assessment of several neurological and psychiatric disorders. Still today EEG is a suitable modality for this purpose due to technical factors and to electrophysiological characteristics. Using group ICA on EEG eyes-closed resting-state in two independent databases we have found seven replicable independent components (IC) describing about $92.5 \%$ of the total EEG variance. The seven components can be grouped into two separate networks (Congedo et al. 2010). The fact that the component spectral profile and spatial localization closely agree in two independent large-sample databases support the notion that they represent true "ground-states" of the resting brain.

The aim of this paper was to describe such resting state pattern and to detail the NICA methodology. This work has included all necessary technical details enabling replication and further use by peers. As compared to scalp EEG normative databases the advantages of NICA are 1) the suppression of feature co-linearity due to the suppression of volume conduction (engendered by ICA) and 2) feature compression. The critical question is whether those advances provide better sensitivity and specificity. It should be stressed that whereas EEG norms are a tool for aiding diagnosis and assessment, they are subjected to false positive and false negatives as per any 
EEG RSN by Group ICA - Congedo et al. 2010

statistical diagnostic tools. Nonetheless, developing on first attempts working in the sensor space, the accuracy can be increased thanks to modern signal processing methods, which was the motivation animating this study. 
EEG RSN by Group ICA - Congedo et al. 2010

\section{Acknowledgements:}

This Research has been partially supported by the French National Research Agency (ANR) within the National Network for Software Technologies (RNTL), project Open-ViBE (“Open Platform for Virtual Brain Environments"), grant \# ANR05RNTL01601. The adult norming studies at BRL were supported in part by grants from National Institute of Aging NIA \#MH32577 and the National Science Foundation \#NS15638. The NTE database was kindly provided by Nova Tech EEG, Inc., Mesa (AZ). 


\section{REFERENCES}

Abraham, A., Schubotz, R.I., von Cramon, D.Y. 2008. Thinking about the future versus the past in personal and non-personal contexts. Brain Res 1233, 106-19.

Ahn, H., Prichep, L.S., John, E.R., Baird, H., Trepetin, M., Kaye, H., 1980. Developmental equations reflect brain dysfunctions. Science 210, 1259-1262.

Asada, H., Fukuda, Y., Tsunoda, S., Yamaguchi, M., Tonoike,. M. 1999. Frontal midline theta rhythms reflect alternative activation of prefrontal cortex and anterior cingulate cortex in humans. Neurosci Lett 274, 29-32.

Auer, D.P., 2008. Spontaneous low-frequency blood oxygenation level-dependent fluctuations and functional connectivity analysis of the 'resting' brain. Magn Reson Imaging, 26, 1055-1064.

Baliki, M.N., Geha, P.Y., Apkarian, A.V., Chialvo, D.R. 2008. Beyond Feeling: Chronic pain hurts the brain, Disrupting the default-mode network dynamics. J Neurosci 28(6), 1398-1403.

Barry, R.J., Clarke, A.R., Johnstone, S.J., Magee, C.A., Rushby, J.A. 2007. EEG differences between eyesclosed and eyes-open resting conditions. Clin Neurophysiol, 118, 2765-2773.

Beckmann, C.F., DeLuca, M., Devlin, J.T., Smith, S.M. 2005. Investigations into resting-state connectivity using independent component analysis. Phil Trans R Soc B 360, 1001-1013.

Behrens, T.E., Woolrich, M.W., Walton, M.E., Rushworth, M.F. 2007. Learning the value of information in an uncertain world. Nat Neurosci 10, 1214-1221.

Berthoz, A. 1997. Parietal and hippocampal contribution to topokinetic and topographic memory. Philos Trans R Soc Lond B Biol Sci 352(1360), 1437-48.

Blanke, O.S., Ortigue S., Landis T., Seeck, M. 2002. Stimulating illusory own-body perceptions. Nature 419(6904), 269-70.

Bloomfield, P. 2000. Fourier analysis of time series. John Wiley \& Sons, New York.

Bluhm, R.L., Osuch, E.A., Lanius, R.A., Boksman, K., Neufeld, R.W.J., Théberge, J. et al. 2008. Default mode network connectivity: effects of age, sex, and analytic approach. Neuroreport 19(8), 887-891.

Brett, M., Johnsrude, I.S., Owen, A.M. 2002. The problem of functional localization in the human brain. Nat Rev Neurosci 3(3), 243-249.

Brillinger, D.R. 1975. Time series: Data analysis and theory. Holt, Rinehart, and Winston, New York. 
Broyd, S.J., Demanuele, C., Debener, S., Helps, S.K., James, C.J., Sonuga-Barke, E.J. 2008. Default-mode brain dysfunction in mental disorders: A systematic review. Neurosci Biobehav Rev 33(3), 279-96.

Buckner, R.L., Andrews-Hanna, J.R., Schacter, D.L. 2008. The brain's default network:, anatomy, function, and relevance to disease. Ann N Y Acad Sci 1124, 1-38.

Calhoun, V.D., Adali, T., Pearlson, G.D., Pekar, J.J. 2001. A method for making group inferences from functional MRI data using independent component analysis. Hum Brain Mapp 14, 140-151.

Castellanos, F.X., Margulies, D.S., Kelly, C., Uddin, L.Q., Ghaffari, M., Kirsch, A. et al. 2008. Cingulateprecuneus interactions: a new locus of dysfunction in adult attention-deficit/hyperactivity disorder. Biol Psychiatry 63, 332-337.

Cavanna, A.E., Trimble, M.R. 2006. The precuneus:, a review of its functional anatomy and behavioural correlates. Brain 129(Pt 3), 564-83.

Chen, A.C., Feng, W., Zhao, H., Yin, Y., Wang, P. 2008. EEG default mode network in the human brain: spectral regional field powers. Neuroimage 41, 561-574.

Coburn, K.A., Lauterbach, E.L, Boutros, N., Kevin, J., Black, M.D., David, B. et al 2006. The value of Quantitative electroencephalography in Clinical Psychiatry. J Neuropsychiatry Clin Neurosci 18: 460-500.

Congedo, M., Gouy-Pailler, C., Jutten, C. 2008. On the blind source separation of human electroencephalogram by approximate joint diagonalization of second order statistics. Clin Neurophysiol 119, 2677-2686.

Congedo, M. 2006. Subspace Projection Filters for Real-Time Brain Electromagnetic Imaging, IEEE Trans Biomed Eng 53(8), 1624-1634.

Congedo, M., John, R.E., De Ridder, D., Prichep, L., Isenhart, B. 2010. On the "dependence” of "independent" group EEG sources; an EEG study on two large databases. Brain Topography, 23(2), 134.

Congedo, M., Lubar, J.F. 2003. Paramatric and non-parametric normative database comparisons in electroenephalography, J Neurother 7(3/4), 1-29.

Congedo, M., Özen, C., Sherlin, L. 2002. Notes on EEG Resampling by Natural Cubic Spline Interpolation. J Neurother 6(4), 73-80.

Congedo, M., Pham, D.-T. 2009. Least-Squares Joint Diagonalization of a matrix set by a congruence transformation, SinFra'09 (Singaporean-French IPAL Symposium), Fusionopolis, Singapore, Feb 18-20. 
Critchley, H.D., 2005. Neural mechanisms of autonomic, affective, and cognitive integration. J Comp Neurol 493, 154-166.

Damoiseaux, J.S., Rombouts, S.A.R.B., Barkhof, F., Scheltens, P., Stam, C.J., Smith, S.M. 2006. Consistent resting-state networks across healthy subjects. Proc Natl Acad Sci U S A 103(37), 13848-13853.

De Ridder, D.K., Van Laere, K., Dupont, P., Menovsky, T., Van de Heyning, P. 2007. Visualizing out-of-body experience in the brain. N Engl J Med 357(18), 1829-33.

Drevets, W.C., Savitz, J., Trimble, M. 2008. The subgenual anterior cingulate cortex in mood disorders. CNS Spectr 13(8), 663-81.

Duffy, F.H., Albert, M.S., McAnulty, G.B. 1993. The pattern of age-related differences in electrophysiological activity of healthy subjects. Neurobiol Aging 14, 73-74.

Eichele, T., Debener, S., Calhoun, V.D., Specht, K., Engel, A.K., Hugdahl, et al. 2008. Prediction of human errors by maladaptive changes in event-related brain networks. Proc Natl Acad Sci U S A. 105(16), 6173-8.

Elliott, R., Friston, K.J., Dolan, R.J., 2000. Dissociable neural responses in human reward systems. J Neurosci 20(16), 6159-65.

Fair, D.A., Cohen, A.L., Desenback, N.U.F., Church, J.A., Miezin, F.M., Barch, D.M. et al. 2007. Development of distinct control networks through segregation and integration, Proc Natl Acad Sci U S A 105(10), 40284032.

Farah, M. J. 1989. The neural basis of mental imagery, Trends Neurosci 12(10), 395-9.

Fein, G., Galin, D., Yingling, C.D., Johnstone, J., Nelson, M.A. 1984. EEG spectra in 9-13 year old boys are stable over 1-3 years. Electroencepgalogr Ciln Neutophysiol 58, 517-518.

Foucher, J.R., Otzenberger, H., Gounot, D. 2003. The BOLD response and the gamma oscillations respond differently than evoked potentials: an interleaved EEG-fMRI study. BMC Neurosci 4(1), 22.

Fox, M.D., Corbetta, M., Snyder, A.Z., Vincent, J.L., Raichle, M.E. 2006. Spontaneous neuronal activity distinguishes human dorsal and ventral attention systems. Proc Natl Acad Sci U S A 103(26), 10046-51.

Fox, M.D., Raichle, M.E. 2007. Spontaneous fluctuations in brain activity observed with functional magnetic resonance imaging. Nat Rev Neurosci 8(9), 700-711. 
Fox, M.D., Snyder, A.Z., Vincent, J.L., Corbetta, M., van Essen, D.C., Raichle, M.E. 2005. The human brain is intrinsically organized into dynamic, anticorrelated functional networks. Proc Natl Acad Sci U S A 102(27), 9673-8.

Fransson, P. 2005. Spontaneous low-frequency BOLD signal fluctuations: an fMRI investigation of the restingstate default mode of brain function hypothesis, Hum Brain Mapp 26(1), 15-29.

Fuchs, M., Kastner, J., Wagner, M., Hawes, S., Ebersole, J.S. 2002. A standardized boundary element method volume conductor model. Clin Neurophysiol 113(5), 702-12.

Garrity, A.G., Pearlsin, G.D., McKiernan, K., Lloyd, D., Kiehl, K.A., Calhoun, V.D. 2007. Aberrant 'deafult mode' functional connectivity in schizophrenia. Am J Psychiatry 164, 450-457.

Ghashghaei, H.T., Hilgetag. C.C., Barbas, H. 2007. Sequence of information processing for emotions based on the anatomic dialogue between prefrontal cortex and amygdala. Neuroimage 34, 905-923.

Goncalves, S.I., de Munck, J.C., Pouwels, P.J.W., Schoonhoven, R., Kujer, J.P.A., Maurits, N.M., et al. 2006. Correlating the alpha rhythm to BOLD using simultaneous EEG/fMRI: inter-subject variability. Neuroimage 30, 203-213.

Grabenhorst, F., Rolls, E.T., Parris, B.A. (2008). From affective value to decision-making in the prefrontal cortex. Eur J Neurosci 28(9), 1930-9.

Greenblatt, R.E., Ossadtchi, A., Pflieger, M.E. 2005. Local Linear Estimators for the Bioelectromagnetic Inverse Problem. IEEE Trans Sig Process 53(9), 3403-3412.

Greicius, M.D., Srivastava, G., Reiss, A.L., Menon, V. 2004. Default more network activity distinguishes Alzheimer's disease from healthy aging: Evidence from functional fMRI, Proc Natl Acad Sci U S A 101, 4637-4642.

Hernández, J.L., Valdés, P., Biscay, R., Virues, T., Szava, S., Bosch, J., et al. 1994. A global scale factor in brain. Int J Neorosci 76, 267-278.

Hughes, J.R., John, E.R. 1999. Conventional and quantitative electroencephalography in psychiatry. J Neuropsychiatry Clin Neurosci 11, 190-208.

Hyvärinen, A., Karhunen, J., Oja, E. 2001. Independent Component Analysis. John Wiley \& Sons, New York. Jann, K., Dierks, T., Boesch, C., Kottlow, M., Strik, W., Koenig, T. 2009. BOLD correlates of EEG alpha phase-locking and the fMRI default mode network. Neuroimage 45(3), 903-916. 
Jasper, H.H. 1958. The ten-twenty electrode system of the International Federation. Electroenceph. Clin. Neurophysiol. 10, 371-375.

John, E.R., Ahn, H., Prichep, L.S., Trepetin, M., Brown, D., Kaye, H. 1980a. Developmental equations for the electroencephalogram. Science 210. 1255-1258.

John, E.R., Karmel, B., Corning, W., Easton, P., Brown, D., Ahn, H., et al. 1977. Neurometrics: Numerical taxonomy identifies different profiles of brain functions within groups of behaviorally similar people. Science $196,1393-1410$.

John, E.R., Karmel, B.Z., Corning, W.C., Easton, P., Brown, D., Ahn, H., et al. 1980b. Neurometrics. Science 196, 1393-1409.

John, E.R., Prichep, L.S. 2009. Principles and applications of quantitative electroencephalogram in psychiatry. In BJ Sadock, VA Sadock, \& P Ruiz (Eds.), Kaplan and Sadock's Comprehensive Textboook of Psychiatry (9th ed., pp. 1013-1032), Lippincott Williams \& Wilkins, Philadelphia.

John, E.R., Prichep, L.S., Easton, P. 1987. Normative data banks and neurometrics, Basic concepts, method and results of norm constructions. In AS Gevins \& A Remond (Eds.), Method of analysis of brain electrical and magnetic signals: Vol. 1. EEG handbook (revised series). Elsevier Science Publishers B.V. (Biomedical Division), New York.

John, E.R., Prichep, L.S., Fridman, J., Easton, P., 1988. Neurometrics: Computer assisted differential diagnosis of brain dysfunctions. Science 239. 162-169.

Johnson, M.R., Mitchell, K.J., Raye, C.L., D'Esposito, M., Johnson, M.K. 2007. A brief thought can modulate activity in extrastriate visual areas: Top-down effects of refreshing just-seen visual stimuli Neuroimage 37(1), $290-9$.

Jurcak, V., Tsuzuki, D., Dan, I. 2007. 10/20, 10/10, and 10/5 systems revisited: their validity as relative headsurface-based positioning systems. Neuroimage 34(4), 1600-1611.

Inanaga, K. 1998. Frontal midline theta rhythm and mental activity. Psychiatry Clin Neurosci 52, 555-566.

Kahn, I., Andrews-Hanna, J.R., Vincent, J.L., Snyder, A.Z., Buckner, R.L. 2008. Distinct cortical anatomy linked to subregions of the medial temporal lobe revealed by intrinsic functional connectivity, J Neurophysiol 100(1), 129-39. 
Keedwell, P.A., Andrew, C., Williams, S.C., Brammer, M.J., Phillips, M.L. 2005. The neural correlates of anhedonia in major depressive disorder. Biol Psychiatry 58(11), 843-53.

Kennedy, D.P., Redcay, E., Courchesne, E. 2006. Failing to deactivate, resting functional abnormalities in autism. Proc Natl Acad Sci U S A 103, 8275-8280.

Kennerley, S.W., Walton, M.E., Behrens, T.E., Buckley, M.J., Rushworth, M.F. 2006. Optimal decision making and the anterior cingulate cortex. Nat Neurosci 9(7), 940-7.

Kondacs, A., Szabo, M. 1999. Long-term inta-individual variability of thebackground EEG in Normals. Clin Neurophysiol 100, 1708-1716.

Kopřivová, J., Congedo, M., Praško, J., Raszka, M., Brunovský, M., Tišlerová, B., Horáček, J., 2009. Medial frontal EEG low-frequency excess in obsessive-compulsive disorder determined by sLORETA and ICA, submitted.

Lachaux, J.P., Fonlupt, P., Kahane, P., Minotti, L., Hoffmann, D., Bertrand, O., Baciu, M. 2007. Relationship between task-related gamma oscillations and BOLD signal: New insights from combined fMRI and intracranial EEG. Hum Brain Mapp. 28(12), 1368-75.

Lancaster, J.L., Woldorff, M.G., Parsons, L.M., Liotti, M., Freitas, C.S., Rainey, L., et al. 2000. Automated talairach atlas labels for functional brain mapping, Human Brain Map 10, 120-131.

Laufs, H. (2008). Endogenous brain oscillations and related networks detected by surface EEG-combined fMRI. Hum Brain Mapp 29(7), 762-9.

Laufs, H., Hammandi, K., Salek-Haddadi, A., Kleinschmidt, A.K., Duncan, J.S., Lemieux, L. 2007. Temporal lobe interictal epileptic discharges affect cerebral activity in 'defaul mode' brain regions. Hum Brain Mapp 28, 1023-1032.

Laufs, H., Krakow, K., Sterzer, P., Eger, E., Bayerle, A., Salek-Haddadi, A., et al. 2003. Electroencephalographic signatures of attentional and cognitive default modes in spontaneous brain activity fluctuations at rest. Proc Natl Acad Sci U S A 100, 11053-11058.

Lopes da Silva, F. 2004. Functional Localization of Brain Sources using EEG and/or MEG data: Volume Conductor and Source Models. Magn Res Img 22, 1533-1538. 
Makeig, S., Debener, S., Onton, J., Delorme, A. 2004 Mining event-related brain dynamics. Trends Cogn Sci. 8(5), 204-10.

Mantini, D., Perrucci, M.G., Del Gratta, C., Romani, G.L., Corbetta, M. 2007. Electrophysiological signatures of resting state networks in the human brain. Proc Natl Acad Sci U S A 104(32), 13170-13175.

Margulies, D.S., Kelly, A.M., Uddin, L.Q., Biswal, B.B., Castellanos, F.X., Milham, M.P. 2007. Mapping the functional connectivity of anterior cingulate cortex. Neuroimage 37, 579-588.

Mazziotta, J., Toga, A., Evans, A., Fox, P., Lancaster, J., Zilles, K., et al 2001. A probabilistic atlas and reference system for the human brain: International Consortium for Brain Mapping (ICBM). Philos Trans R Soc Lond B Biol Sci 356(1412), 1293-1322.

Mottaghy, F.M., Willmes, K., Horwitz, B., Muller, H.W., Krause, B.J., Sturm, W. 2006. Systems level modeling of a neuronal network subserving intrinsic alertness. Neuroimage 29, 225-233.

Morcom, A.M., Fletcher, P.C. 2007. Does the brain have a baseline? Why we should be resisting a rest. Neuroimage 37(4), 1073-82.

Mukamel, R., Gelbard, H., Arieli, A., Hasson, U., Fried, I., Malach, R. 2005. Coupling between neuronal firing, field potentials, and FMRI in human auditory cortex. Science 309(5736), 951-4.

Nir, Y., Fisch, L., Mukamel, R., Gelbard-Sagiv, H., Arieli, A., Fried, I., Malach, R. 2007. Coupling between neuronal firing rate, gamma LFP, and BOLD fMRI is related to interneuronal correlations. Curr Biol 17(15), 1275-85.

Nunez, P.L., Srinivasan, R. 2006. Electric Field of the Brain, 2nd ed., Oxford Univ Press, New York.

Nuwer, M.R., 1988. Quantitative EEG: II. Frequency analysis and topographic mapping in clinical settings. J of Clin Neurophysiology 5, 45-85.

Onton, J., Delorme, A., Makeig, S. 2005. Frontal midline EEG dynamics during working memory. Neuroimage, $27,341-356$.

Oken, B.S., Chiappa, K.H. 1988. Short-term variability in EEG frequency analysis. Electroencephalogr Clin Neurophysiology 69, 191-198.

Pascual-Marqui, R.D. 2002. Standardized Low Resolution brain electromagnetic Tomography (sLORETA): technical details. Methods Find Exp Clin Pharmacol 24D, 5-12. 
Pascual-Marqui, R.D. 2007. Discrete, 3D distributed, linear imaging methods of electric neuronal activity. Part 1: exact, zero error localization, arXiv, 0710.3341.

Pham, D.-T. 2001. Blind separation of instantaneous mixture of sources via the Gaussian mutual information criterion. Signal Process 81, 855-70.

Pham, D.-T., Congedo, M. 2009. Least square joint diagonalization of matrices under an intrinsic scale constraint, ICA 2009 ( $8^{\text {th }}$ International Conference on Independent Component Analysis and Signal Separation, March 15-18, Paraty, Brasil, 298-305.

Pellijeff, A., Bonilha, L., Morgan, P.S., McKenzie, K., Jackson, S.R. 2006. Parietal updating of limb posture: an event-related fMRI study. Neuropsychologia 44(13), 2685-90.

Prichep, L.S. 2005. Use of normative databases and statistical methods in demonstrating clinical utility of QEEG: Importance and cautions . Clinical EEG 36: 82-7.

Raichle, M.E., Mintum, M.A. 2006. Brain work and brain imaging. Annu Rev Neurosci 29, 449-476.

Raichle, M.E., Snyder, A.Z. 2007. A default mode of brain function: A brief history of an evolving idea. Neuroimage 37, 1083-1090.

Richer, F., Martinez, M., Robert, M., Bouvier, G., Saint-Hilaire, J.M. 1993. Stimulation of human somatosensory cortex: tactile and body displacement perceptions in medial regions. Exp Brain Res 93(1), 1736.

Rilling, J.K., Barks, S.K., Parr, L.A., Preuss, T.M., Tracy, L.F., Pagnoni, G., et al. 2007. A comparison of resting state brain activity in humans and chimpanzees. Proc Natl Acad Sci U S A 104(43), 17146-17151.

Rogers, R.D., Ramnani, N., Mackay, C., Wilson, J.L., Jezzard, P., Carter, C.S., Smith, S.M. 2004. Distinct portions of anterior cingulate cortex and medial prefrontal cortex are activated by reward processing in separable phases of decision-making cognition. Biol Psychiatry 55(6), 594-602.

Rombouts, S.A., Barkhof, F., Goekoop, R., Stam, C.J., Scheltens, P. 2005. Altered resting state networks in mild cognitive impairment and mild Alzheimer's disease: an fMRI study. Hum Brain Mapp 26, 231-239.

Sammer, G., Blecker, C., Gebhardt, H., Bischoff, M., Stark, R., Morgen, K., Vaitl, D. 2007. Relationship between regional hemodynamic activity and simultaneously recorded EEG-theta associated with mental arithmetic-induced workload. Hum Brain Mapp 28(8), 793-803. 
Scheeringa, R., Bastiaansen, M.C.M., Petersson, K.M., Oostenveld, R., Norris, D.G., Hagoort, P. 2008. Frontal theta EEG activity correlates negatively with the default mode network in resting state. Int J Psychophysiol 67, 242-251.

Schmithorst, V.J., Holland, S.K. 2004. Comparison of three methods for generating group statistical inferences from independent component analysis of functional magnetic resonance imaging data. Magn Reson Imaging 19(3), 365-368.

Schreckenberger, M., Lange-Asschenfeldt, C., Lochmann, M., Mann, K., Siessmeier, T., Buchholz, H.G., et al. 2004. The thalamus as the generator and modulator of EEG alpha rhythm: a combined PET/EEG study with lorazepam challenge in humans. Neuroimage 22, 637-644.

Seeley, W.W., Menon, V., Schatzberg, A.F., Keller, J., Glover, G.H., Kenna, H., et al. 2007. Dissociable intrinsic connectivity networks for salience processing and executive control. J Neurosci 27, 2349-2356.

Sorg, C., Riedl, V., Mühlau, M., Calhoun, V.D., Eichele, T., Läer, L., et al. 2007. Selective changes of restingstate networks in individuals at risk for Alzheimer's disease. Proc Natl Acad Sci U S A. 104(47), 18760-5.

Sridharan, D., Levitin, D.J., Menon, V. 2008. A critical role for the right fronto-insular cortex in switching between central-executive and default-mode networks, Proc Natl Acad Sci U S A 105(34), 12569-74.

Stein J.L., Wiedholz L.M., Bassett D.S., Weinberger D.R., Zink C.F., Mattay V.S. et al. 2007. A validated network of effective amygdala connectivity. Neuroimage 36(3), 736-45.

Stephan, T., Deutschländer, A., Nolte, A., Schneider, E., Wiesmann, M., Brandt, T., Dieterich, M. 2005. Functional MRI of galvanic vestibular stimulation with alternating currents at different frequencies. Neuroimage 26(3), 721-32.

Svoboda, E., McKinnon, M.C., Levine, B. 2006. The functional neuroanatomy of autobiographical memory: a meta-analysis, Neuropsychologia 44(12), 2189-208.

Talairach, J., Tournoux, P., 1988. Co-planar stereotaxic atlas of the human brain. Thieme Medical Publishers, New York.

Terakawa, Y., Tsuyuguchi, N., Tanaka, H., Shigihara, Y., Sakamoto, S., Takami, T., et al. 2008. Quantitative analysis of MEG using modified sLORETA for clinical application. Clin Neurophysiol 119 (8), 1917-1922.

Tichavsky, P. Yeredor, A. 2009. Fast Approximate Joint Diagonalization Incorporating Weight Matrices. IEEE Trans Sig Process 57(3), 878-891. 
Uddin, L.Q., Kelly, A.M., Biswal, B.B., Margulies, D.S., Shehzad, Z., Shaw, D., et al. 2008. Network homogeneity reveals decreased integrity of default-mode network in ADHD. J Neurosci Methods 169(1), 24954.

van Beijsterveldt, C.E.M., Molenaar, P.C.M., de Gaus, E.J.C., Boosma, D.I. 1996. Heritability of human brain functioning as assessed by electroencephalography. Am J Hum Genet 58, 562-573.

van den Heuvel, M., Mandl, R., Hulshoff Pol, H. 2008. Normalized cut group clustering of resting-state FMRI data. PLoS ONE 3(4), e2001.

van der Loo, E., Gais, S., Congedo, M., Vanneste, S., Plazier, M., Menovsky, T., Van de Heyning, P., De Ridder, D. 2009. Tinnitus intensity dependent gamma oscillations of the contralateral auditory cortex. PLoS One. 2009 Oct 9;4(10),e7396.

van Essen, D.C. 2005. A Population-Average, Landmark- and Surface-based (PALS) atlas of human cerebral cortex. Neuroimage 28(3), 635-62.

Vincent, J.L., Patel, G.H., Fox, M.D., Snyder, A.Z., Baker, J.T. 2007. Intrinsic functional architecture in the anesthesized monkey brain. Nature 447, 83-86.

Wagner, M., Fuchs, M., Kastner, J. 2004. Evaluation of sLORETA in the presence of noise and multiple sources. Brain Topogr 16(4), 277-280.

Wang, K., Jiang, T., Yu, C., Tian, L., Li, J., Liu, Y., Zhou, Y., Xu, L., Song, M., Li, K. 2008. Spontaneous activity associated with primary visual cortex: a resting-state FMRI study, Cereb Cortex 18(3), 697-704.

Wax, M., Kailath, T. 1985. Detection of Signals by Information Theoretic Criteria. IEEE Trans Acoust 33(2), 387-392.

Welch, P.D. 1967. The Use of Fast Fourier Transform for the Estimaton of Power Spectra: A Method Based on Time Averaging Over Short, Modified Periodograms. IEEE Trans Audio Electroacoustics 15(2), 70-74. 


\section{Figures}

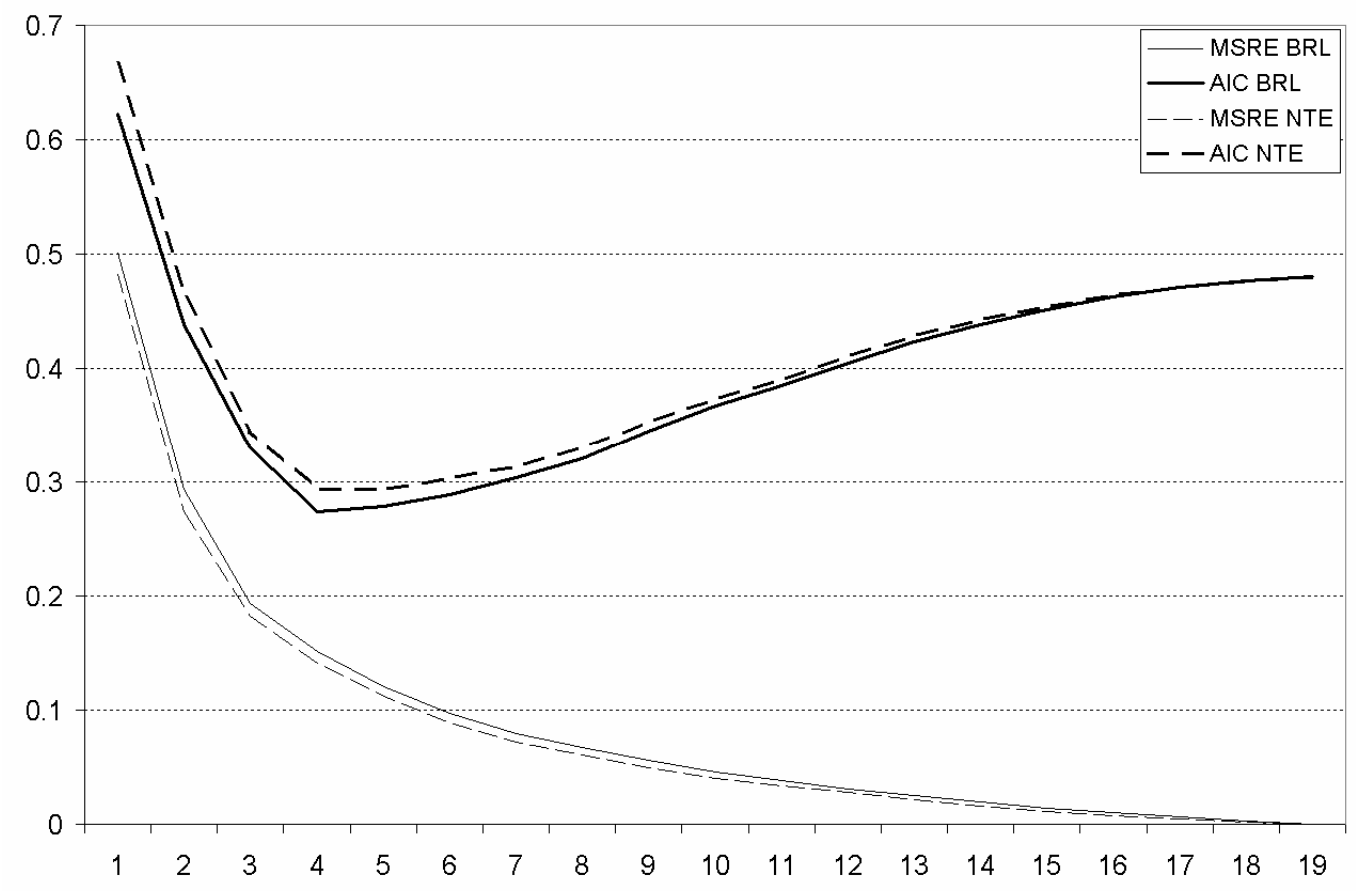

Figure 1 : The eigenstructure of the grand-agverage covariance matrix in the $0.5-30 \mathrm{~Hz}$ range is very similar in the two databases (BRL and NTE), as seen by the mean square representation error (MSRE) and Akaike information criterion (AIC) associated to the 19 eigenvalues. The MSRE curves are normalized so to sum up to unity. The AIC is shown in arbitrary units. 
c1
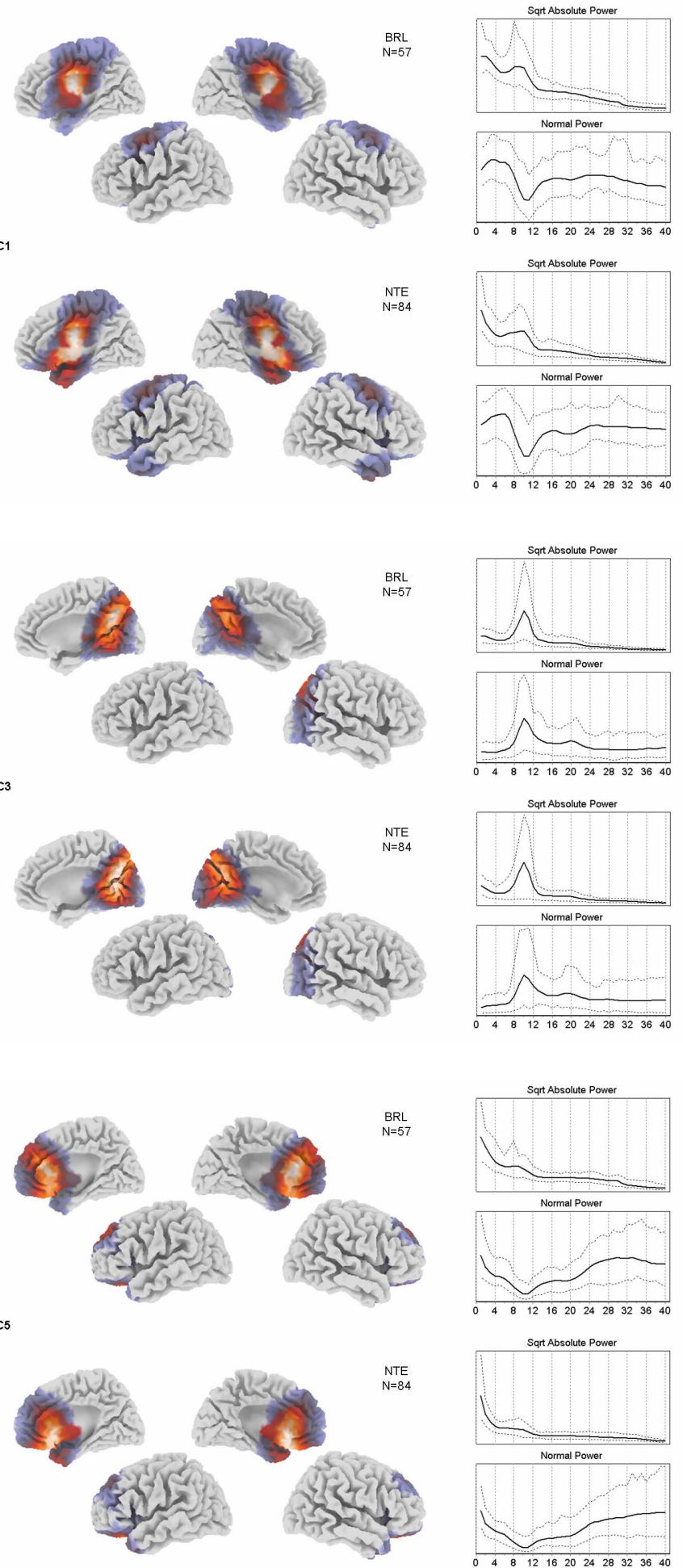
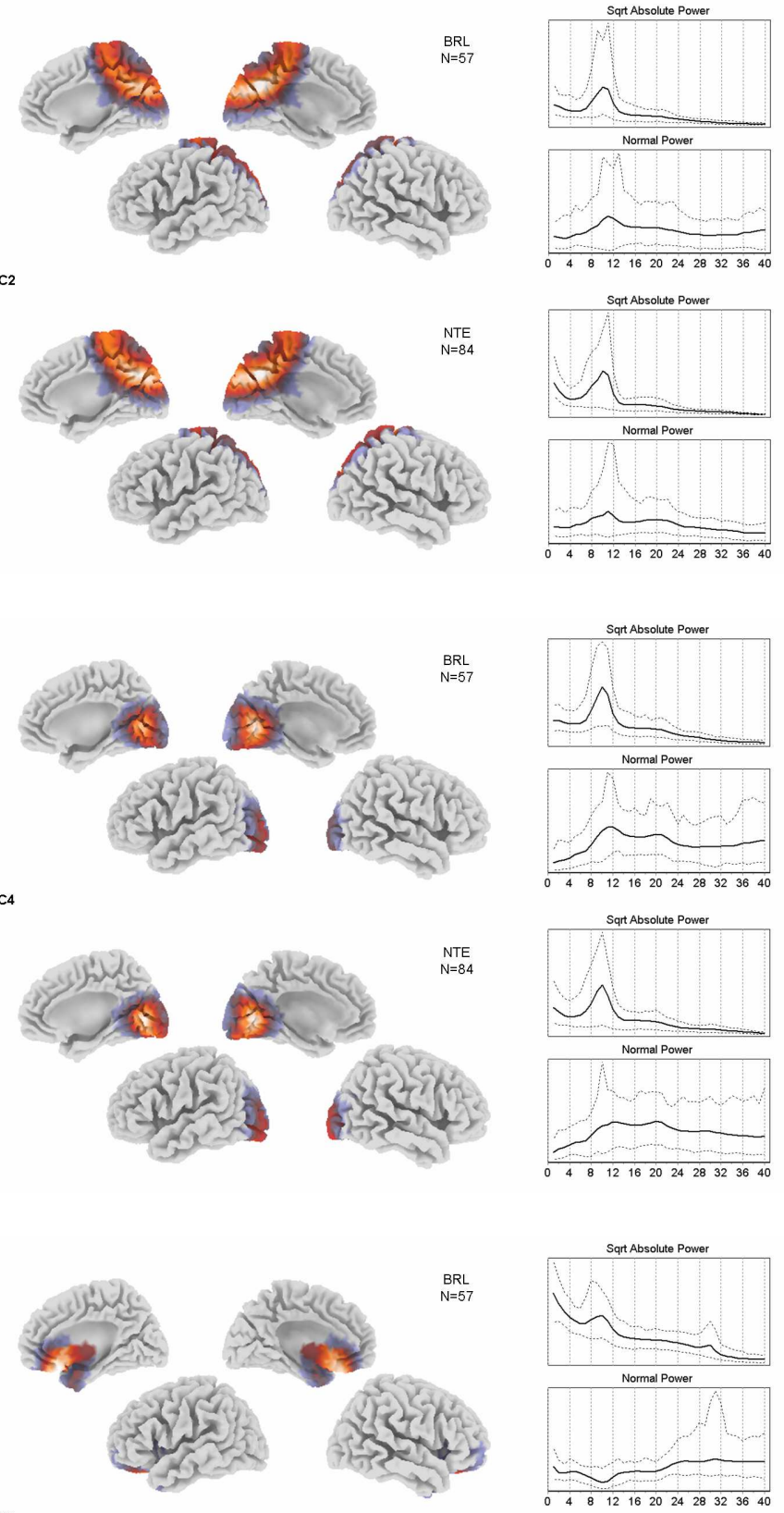

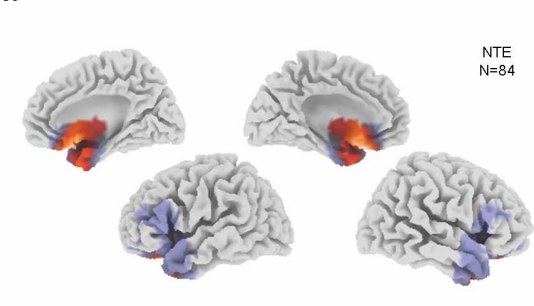

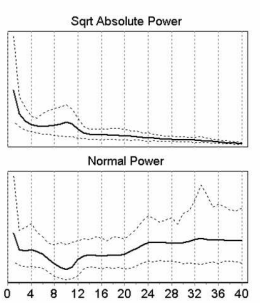




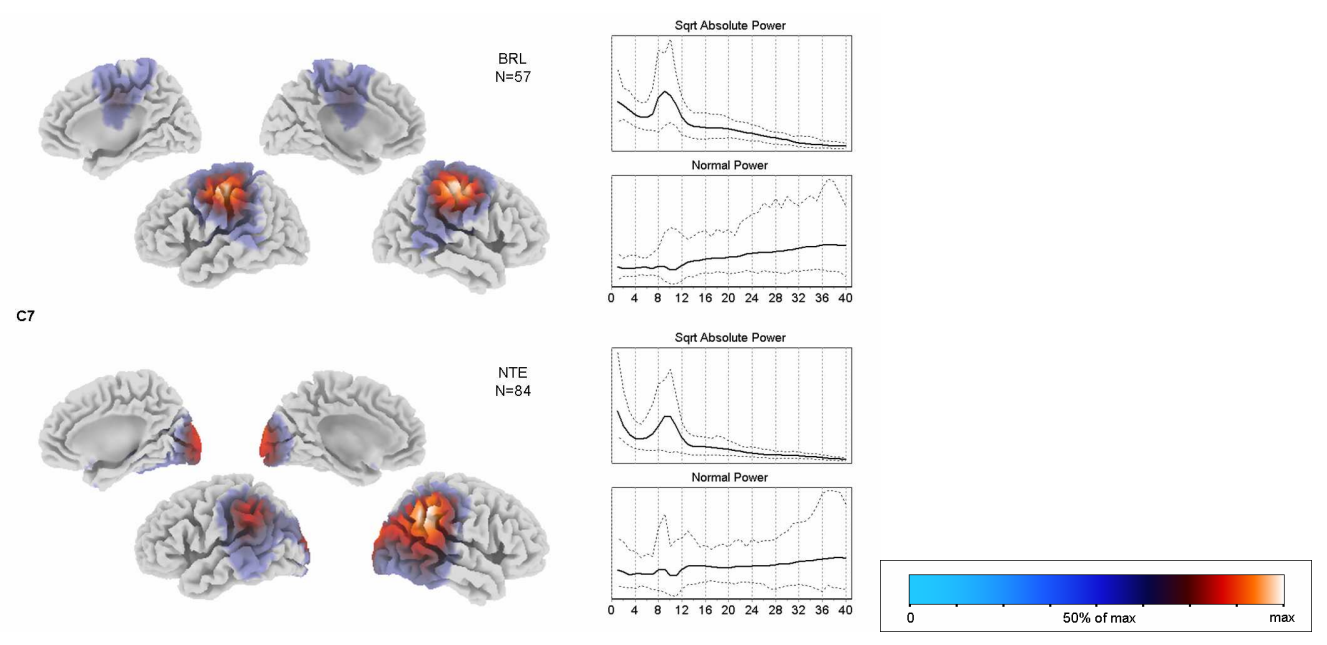

Figure 2. sLORETA cortical current density images and associated frequency spectrum of the seven independent components for the BRL (top) and NTE (bottom) database. From left to right:

- the sLORETA cortical image medial and lateral views of the left and right hemisphere. The current density is thresholded at half the maximum. The anatomical image is based on the CARET software (van Essen, 2005).

- the mean (solid line) and 95\% confidence interval (dotted line) of the grand-average frequency spectrum in the range $0.5-40 \mathrm{~Hz}$ for absolute and normal power. The vertical axis is adjusted individually in each plot. Cortical images have been produced by the sLORETA-Key software. 
EEG RSN by Group ICA - Congedo et al. 2010

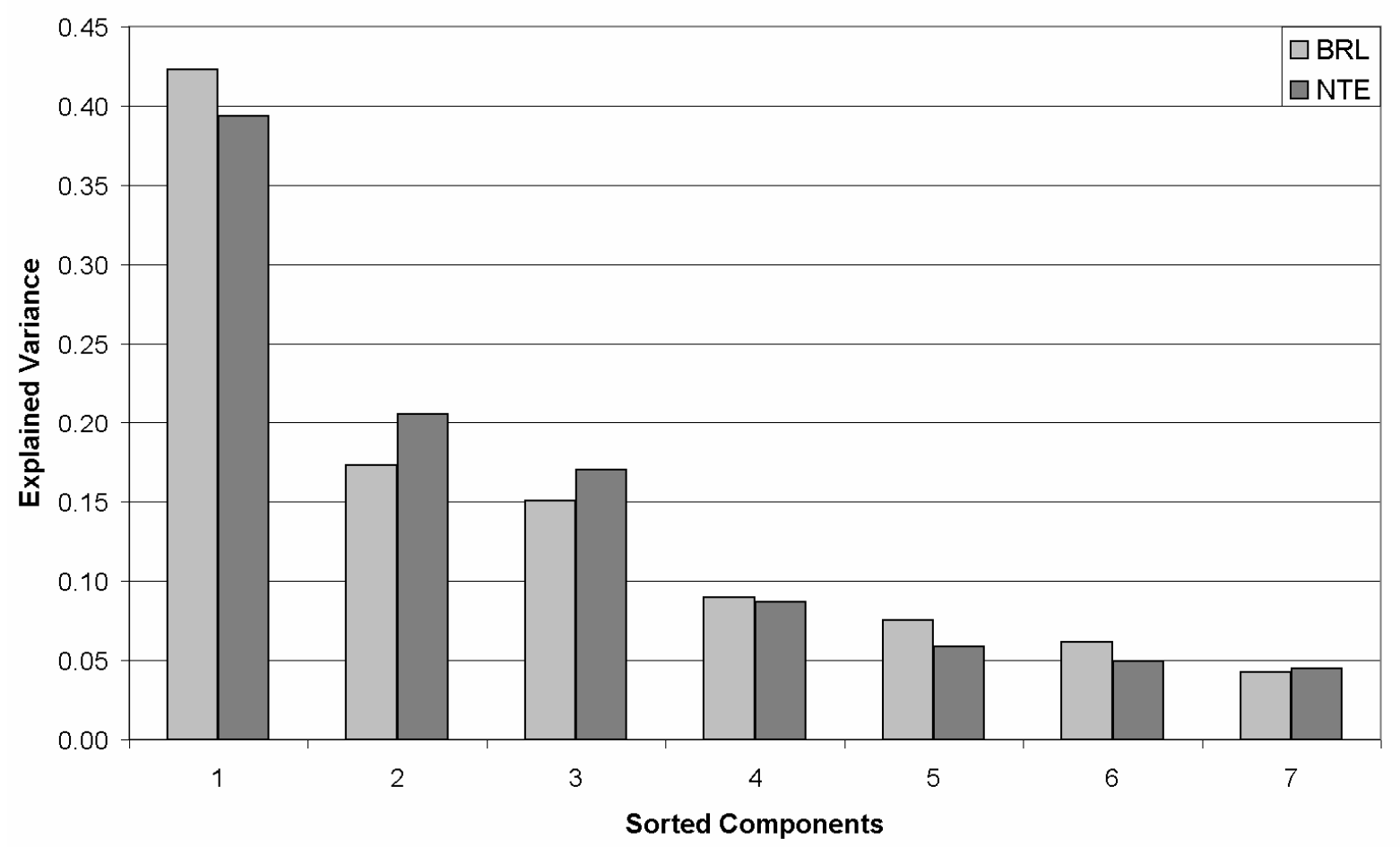

Figure 3: Cumulative proportion of the grand-average variance explained by the seven group ICA components found independently on the NTE and BRL databases. 
Table 1: Anatomical structures and Brodmann areas (BAs) where high-power (more then 50\% of the maximum) current source is located for the seven ICA components in the two databases (leftmost column). BA of top 10\% current density power are highlighted in bold. When the side is not specified labels and corresponding BAs apply bilaterally.

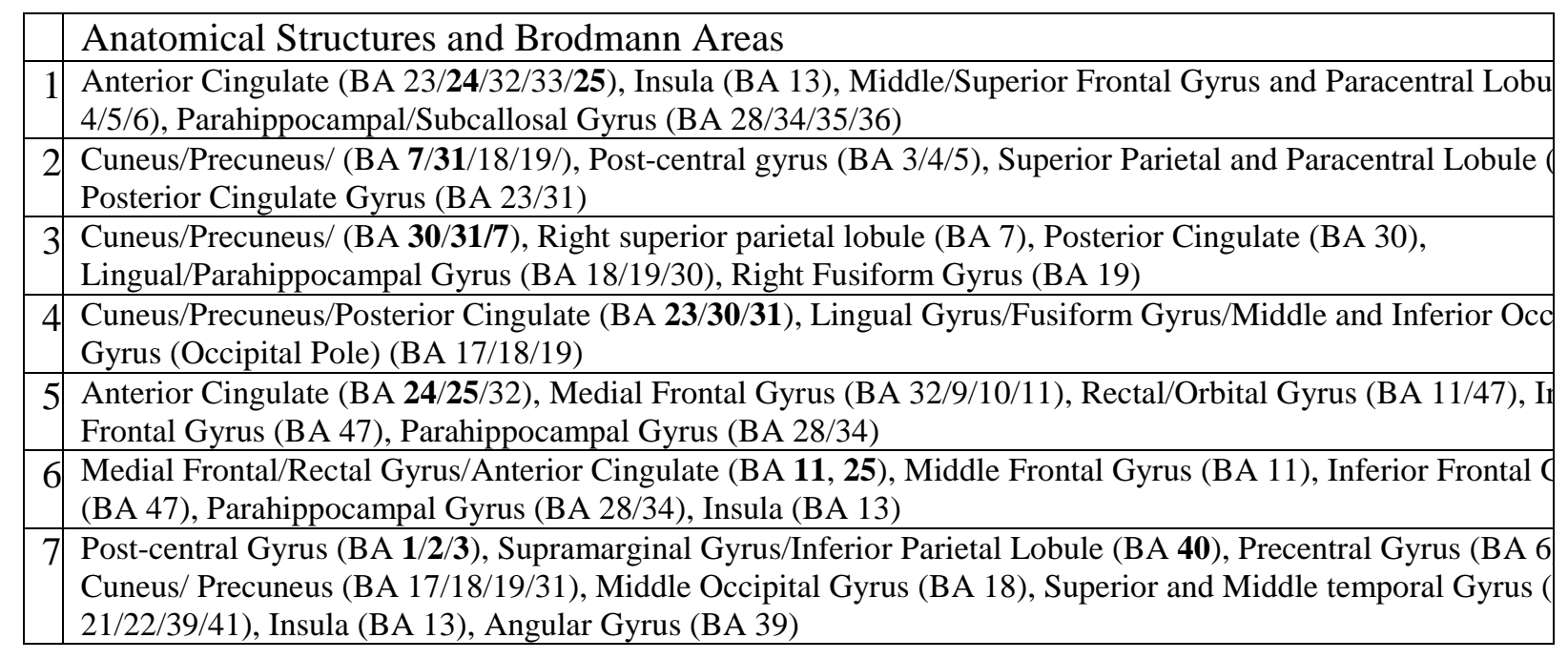

